Wie richtig Stefan Korioth mit seiner Einschätzung liegt, dass eine Fülle neuer und dazu unklarer Mischtatbestände geschaffen worden ist, obwohl die Reform als ausdrückliches Ziel und auch als voreilig verkündeten Erfolg die Entflechtung der Ebenen für sich in Anspruch genommen hat, zeigt der Beitrag von Uwe-Dietmar Berlit. Die Unterkunftskostenerstattung nach $\$ 46$ SGB II (Hartz IV) ist ein Mischfinanzierungstatbestand, der in dieser Größenordnung nur noch mit der Einigung über die Finanzierung des Ausbaus von Kindertagesstätten zu vergleichen ist - beides übrigens Gesetze, die nach Inkrafttreten der Föderalismusreform verabschiedet wurden.

Den Herausgebern ist ein interessanter und wichtiger Beitrag zur laufenden Föderalismusdebatte gelungen. Er versteht es, deutlich zu machen, wie dringlich und groß die Aufgabe ist. Ebenso weist er darauf hin, dass man sich nicht vorschnell mit einem Ergebnis zufrieden geben sollte.

Abschließend sei noch eine kritische Bemerkung gemacht: Wenn es um den Finanzausgleich geht, darf der Hinweis auf „die Herstellung gleichwertiger Lebensverhältnisse im Bundesgebiet“ (Art. 72 Abs. 2 GG) nicht fehlen. Diese Verfassungsnorm wird in einigen Beiträgen als Anspruchgrundlage für das bestehende hohe Ausgleichsniveau interpretiert, vorzugsweise und verständlicherweise von Vertretern der finanz- und wirtschaftsschwachen Länder in Nord- und besonders Ostdeutschland. Die „Herstellung gleichwertiger Lebensverhältnisse" ist aber keine verfassungsrechtliche Anspruchs-, sondern eine Ermächtigungsnorm, die Gesetzgebungskompetenzen verleiht. Das lässt sich nicht zuletzt daraus erschließen, dass sie im Teil über die Gesetzgebung des Bundes steht. Sie verpflichtet den Bund nicht rechtlich, auf dieses Ziel hinzuwirken, sondern sie ermächtigt ihn zu einer bundeseinheitlichen Gesetzgebung, wenn die Voraussetzungen erfüllt sind. Würden sich die Akteure diese Sichtweise zueigen machen, gäbe es sicher einen größeren Reformspielraum. Zu erwarten ist das nicht, weil die Mehrheit der finanzschwachen Länder verständlicherweise jedes Argument zu nutzen versucht, um ein hohes Ausgleichsniveau zu begründen.

Stefan Schieren

\title{
Große dogmatische Darstellung der direkten Demokratie in den Bundesländern
}

\section{Rux, Johannes: Direkte Demokratie in Deutschland. Rechtsgrundlagen und Rechtswirklichkeit der unmittelbaren Demokratie in der Bundesrepublik Deutschland und ihren Ländern, Nomos Verlagsgesellschaft, Baden-Baden 2008, 984 Seiten, € 158,-.}

Den Kern dieser Tübinger juristischen Habilitationsschrift bildet die Untersuchung der direkten Demokratie in den 16 deutschen Bundesländern. Dazu analysiert Johannes Rux zunächst die jeweiligen Landesverfassungen dogmatisch - didaktisch aufgeteilt in zwei Staffeln: die älteren und die neueren Verfassungen - und betrachtet dann eingehend die Rechtswirklichkeit anhand der konkreten Fälle. Bereits die Quantität dieser Darstellung ist beeindruckend. Am Beispiel Schleswig-Holstein: Auf 42 Seiten mit den Rechtsgrundlagen folgen 23 Seiten für die Staatspraxis und verfassungspolitische Bewertung. Dabei behandelt der Autor mit einer immensen Mühe so gut wie jede rechtliche Frage, die sich bei den direktde- 
mokratischen Verfahren bisher stellte oder künftig noch stellen könnte. Wenn man bedenkt, dass die üblichen Landesverfassungskommentare nur ein paar Seiten für die einschlägigen Artikel aufwenden (von der Unsitte der eher paraphrasierenden „Kommentierung “ ganz zu schweigen), dann liegt hier ein systematischer Gesamtkommentar des einschlägigen Landesverfassungsrechts vor, der die Beschäftigung mit dieser Materie auf ein neues Niveau hebt.

Den beiden Staffeln vorangestellt sind zusammenfassende Darstellungen der Verfahren, 72 beziehungsweise 35 Seiten lang, in denen Rux den intraföderalen Vergleich fruchtbar macht. Zusätzlich behandelt er in einem historischen Teil von 92 Seiten die Entwicklung der direkten Demokratie in Deutschland von 1919 bis 1945 und erörtert auf weiteren 58 Seiten das Verhältnis von „Direkter Demokratie und Grundgesetz“. Allem vorausgeschickt aber ist ein einleitender Teil zu „Einführung und Grundlagen“, in dem Rux neben begrifflichen Klärungen und der Eingrenzung des Untersuchungsgegenstandes (kommunale Direktdemokratie ist ausgeklammert) den Sinn dieser Verfahren erörtert und sich mit den Standard-Einwänden auseinandersetzt.

Direktdemokratische Verfahren sollen - so Rux - die strukturellen Defizite eines repräsentativen Systems der politischen Willensbildung zumindest teilweise ausgleichen, und zwar auf zweierlei Weise: Als Kommunikationsinstrument sollen sie auch kleinen Gruppen die Möglichkeit geben, ein bestimmtes Anliegen gegenüber dem Parlament und der Regierung zu artikulieren, und diese verpflichten, sich damit auseinanderzusetzen. Gelingt dabei keine Verständigung, komme ihre zweite - historisch ältere - Funktion zum Tragen: die eines Korrektivs der Regierungspolitik. Erst 1990 habe mit der Verfassungsreform in Schleswig-Holstein ein Paradigmenwechsel eingesetzt. Beide Funktionen seien machttechnisch miteinander verknüpft: Das kommunikative Potential der direktdemokratischen Verfahren hänge entscheidend davon ab, ob eine realistische Chance dafür besteht, dass ein auf dem Wege des Volksbegehrens eingebrachter Entwurf tatsächlich zum Volksentscheid gelangt und dabei auch angenommen wird. Die Parlamente seien nämlich nur dann bereit, sich mit einem „von unten“ vorgebrachten Anliegen mehr als unbedingt nötig auseinanderzusetzen, „wenn sie befürchten müssen, dass ihnen andernfalls die Entscheidung aus der Hand genommen wird“ (S. 397 f.).

Vor diesem theoretischen Hintergrund fällt Rux' Urteil über die Regelungen der älteren Landesverfassungen vernichtend aus: Wegen der extremen Anforderungen sei es den Bürgern faktisch unmöglich, Entscheidungen des Parlaments durch einen Volksentscheid zu korrigieren. Damit laufe aber auch die Artikulations- und Kommunikationsfunktion der direktdemokratischen Verfahren leer. Ihre politische Bedeutung sei daher zu vernachlässigen. Allein in Bayern seien diese Verfahren mehr „als ein bloßes plebiszitäres Placebo“ (S. 404).

Während diese Einschätzung nicht verwundert, kommt Rux'skeptische Bewertung der Regelungen in den neueren Landesverfassungen, einige hundert Seiten weiter, nicht ganz so erwartet: Nach der Zahl der durchgeführten oder zumindest vorbereiteten Verfahren und auch mit Blick darauf, wie oft sich die Initiatoren mit ihren Anliegen zumindest teilweise durchsetzen konnten, scheine die Nutzung der direktdemokratischen Verfahren nun wesentlich einfacher geworden zu sein. Aber dieser Schein trüge. Auch nach den neuen Regelwerken könnten die Initiatoren „kaum damit rechnen“, auch nur die Hürden des Volksbegehrens zu überwinden. Und „selbst wenn dies gelingen sollte, besteht nur eine sehr geringe Chance, dass der Antrag beim Volksentscheid von einer hinreichenden Mehrheit der 
Stimmberechtigten angenommen wird“ (S. 894). Wegen dieser zu geringen Chance der Korrektur sei auch die Funktion als Instrument zur Artikulation und zur Verbesserung der Kommunikation zwischen den Bürgern und ihren Repräsentanten schwer beeinträchtigt. Insgesamt habe die direkte Demokratie in den Bundesländern trotz eines regelrechten Booms in den vergangenen 15 Jahren die mit ihr „verbundenen Hoffnungen bisher nur ansatzweise erfüllt“ (S. 906).

Direkte Demokratie ist, wie Rux zutreffend sieht, in hohem Maße verfahrenssensibel. Bei der Aufgabe, daraus praktikable Instrumente der politischen Willensbildung zu machen, seien die Verfassungs- und Gesetzgeber in den Ländern „bisher auf halbem Wege stehen geblieben“ (S. 908). Daher bleibe „die entscheidende Frage“, ob die direktdemokratischen Verfahren einen maßgeblichen Beitrag zum Ausgleich der strukturellen Defizite des repräsentativ-parlamentarischen Systems leisten könnten, „weiterhin offen“ (S. 908). Um jene Instrumente praktisch handhabbar zu machen, müssten die Hürden auf dem Weg zum Volksentscheid gesenkt, die Tabubereiche zusammengestrichen und schließlich solche Verfahren auch auf der Bundesebene eingeführt werden. Abschließend unterbreitet Rux deshalb einige Vorschläge für eine Optimierung der Verfahren in den Ländern und diskutiert die wichtigsten Probleme, die sich bei der Einführung im Bund stellen.

Der Autor ist ein unabhängiger Kopf, der niemanden schont, wenn er Kritik für geboten hält. So vertritt er strikt die Position, dass die Ausführungsgesetze nur die Vorgaben der Verfassungen zu konkretisieren haben, aber nicht selbständig - wie üblich - zum Beispiel zusätzliche Verfahrenshürden aufrichten, umfassende präventive Normenkontrollen vorschreiben oder Wiederholungssperren einführen dürfen. Umgekehrt haben auch direktdemokratische Mythen bei ihm keine Chance: Die neuerdings favorisierte Zusammenlegung von Volksentscheiden mit Wahlen kritisiert Rux als eine „Manipulation der Beteiligungsquote“ (S. 776) und stellt klar, dass „eine möglichst hohe Abstimmungsbeteiligung keine Gewähr für die Richtigkeit des Abstimmungsergebnisses bietet“ (S. 901), wenngleich er natürlich auch sieht, dass diese Kombinationsidee mit der deutschen Quorensituation zusammenhängt.

Schwächen hat dieses beeindruckende Werk kaum, und die wenigen erklären sich aus der schwierigen Entstehungsgeschichte der umfangreichen Untersuchung, die das Vorwort andeutet. So wurde der 2005 erschienene Sammelband von Andreas Kost über „Direkte Demokratie in den deutschen Ländern“ nicht mehr vollständig ausgewertet. Folge: Bei der Untersuchung der Kostenerstattung nach den neueren Regelwerken stellt Rux nur die Rechtsgrundlagen zusammen (S. 428 f.) und wirft keinen Blick mehr auf die Rechtswirklichkeit, obwohl in jenem Sammelband bereits empirische Daten zu prominenten Fällen gegeben und Kostendeckungsgrade ermittelt worden waren, zu denen man sich die Schärfe der Ruxschen Kommentierung gewünscht hätte.

Insgesamt aber liegt nach der großen Geschichte der direkten Demokratie in Deutschland, die wir Hanns-Jürgen Wiegand verdanken ${ }^{1}$, nun erfreulicherweise auch eine große dogmatische Darstellung der direkten Demokratie in den Bundesländern vor.

Otmar Jung

1 Vgl. Otmar Jung, Eine große Geschichte der direkten Demokratie in Deutschland, in: ZParl, 39. Jg. (2008), H. 1, S. 187 - 189. 